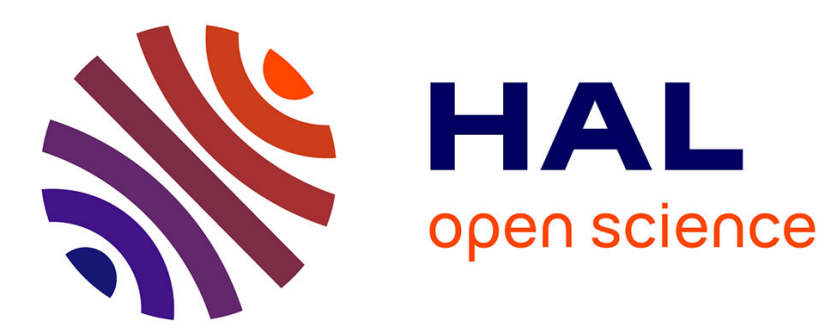

\title{
Étude du ralentissement de l'action dans la maladie de Parkinson au moyen d'un paradigme de chronométrie informatisée
}

Anaïs Élinani

\section{- To cite this version:}

Anaïs Élinani. Étude du ralentissement de l'action dans la maladie de Parkinson au moyen d'un paradigme de chronométrie informatisée. Médecine humaine et pathologie. 2015. dumas-01233658

\section{HAL Id: dumas-01233658 https://dumas.ccsd.cnrs.fr/dumas-01233658}

Submitted on 25 Nov 2015

HAL is a multi-disciplinary open access archive for the deposit and dissemination of scientific research documents, whether they are published or not. The documents may come from teaching and research institutions in France or abroad, or from public or private research centers.
L'archive ouverte pluridisciplinaire HAL, est destinée au dépôt et à la diffusion de documents scientifiques de niveau recherche, publiés ou non, émanant des établissements d'enseignement et de recherche français ou étrangers, des laboratoires publics ou privés. 
Université de Picardie Jules Verne

Faculté médecine Amiens

Année universitaire 2014-2015

\title{
ÉTUDE DU RALENTISSEMENT DE L'ACTION DANS LA MALADIE DE PARKINSON AU MOYEN D'UN PARADIGME DE CHRONOMÉTRIE INFORMATISÉE
}

\author{
THÈSE \\ POUR LE DIPLOME D’ÉTAT DE DOCTEUR EN MÉDECINE \\ SPÉCIALITÉ NEUROLOGIE
}

Présentée et soutenue publiquement le 30 septembre 2015 par

\section{Anaïs ELINANI}

Président du jury : $\quad$ Monsieur le Professeur O. GODEFROY

Membres du jury : $\quad$ Monsieur le Professeur JM. MACRON

Monsieur le Professeur P. KRYSTKOWIAK

Madame le Docteur M. TIR

Madame Martine Roussel

Directeur de Thèse : $\quad$ Monsieur le Professeur P. KRYSTKOWIAK 
Université de Picardie Jules Verne

Faculté médecine Amiens

Année universitaire 2014-2015

\title{
ÉTUDE DU RALENTISSEMENT DE L'ACTION DANS LA MALADIE DE PARKINSON AU MOYEN D'UN PARADIGME DE CHRONOMÉTRIE INFORMATISÉE
}

\author{
THÈSE \\ POUR LE DIPLOME D’ÉTAT DE DOCTEUR EN MÉDECINE \\ Présentée et soutenue publiquement le 30 septembre 2015 par
}

\section{Anaïs ELINANI}
Président du jury : $\quad$ Monsieur le Professeur O. GODEFROY
Membres du jury : $\quad$ Monsieur le Professeur JM. MACRON
Monsieur le Professeur P. KRYSTKOWIAK
Madame le Docteur M. TIR
Madame Martine Roussel

Directeur de Thèse : $\quad$ Monsieur le Professeur P. KRYSTKOWIAK 
Monsieur le Professeur Olivier GODEFROY

Professeur des Universités-Praticien Hospitalier

Chef du service de Neurologie d'Amiens

Pôle « autonomie »

Monsieur le Professeur,

Vous me faites l'honneur de présider cette thèse. Je vous remercie pour l'intérêt que vous avez porté à ce travail, pour votre enseignement et votre expérience dont j'ai pu bénéficier au cours de ces années. Recevez ici toute ma reconnaissance et l'expression de mon plus profond respect. 
Monsieur le Professeur Jean-Michel MACRON

Professeur des Universités-Praticien Hospitalier

(Physiologie)

Chef du Service Explorations Fonctionnelles du Système Nerveux

Pôle Autonomie

Monsieur le Professeur,

Je vous remercie de me faire l'honneur de participer à ce jury. Veuillez recevoir l'expression de ma sincère reconnaissance et de mon profond respect. 
Monsieur le Professeur Pierre KRYSTKOWIAK

Professeur des Universités-Praticien Hospitalier

(Neurologie)

Assesseur du $2^{\text {ème }}$ cycle

Monsieur le Professeur,

Vous m'avez fait l'honneur de diriger cette thèse et je vous en remercie. Je vous remercie également pour la grande qualité de votre enseignement et pour votre expérience dont j'ai pu bénéficier au cours de ma formation. Veuillez trouver ici l'expression de mes remerciements et de mon profond respect. 
Madame le Docteur Mélissa TIR

Praticien Hospitalier

(Neurologie)

Mélissa,

Je vous remercie de me faire l'honneur de participer à ce jury. Je vous remercie aussi chaleureusement pour m'avoir guidée dans les différentes étapes de ce travail, pour vos précieux conseils, votre gentillesse, et aussi pour l'enseignement que vous m'avez apporté durant ces années de formation. Recevez ici toute ma reconnaissance et mon profond respect. 
Madame le Docteur Martine ROUSSEL

Docteur en Neuropsychologie

Chère Madame,

Je vous remercie de me faire l'honneur de participer à ce jury. Et encore un grand merci pour votre aide très précieuse à chaque étape de cette thèse, pour votre expérience, et vos conseils toujours avisés. Je vous suis très reconnaissante également pour votre disponibilité et votre réactivité qui m'ont permis de mener à bien ce travail Recevez ici toute ma gratitude, et mon profond respect. 
Je remercie très chaleureusement le Docteur Cécile Duru, qui a initié ce travail lui construisant de solides bases. Et je la remercie également pour ses bons conseils, son soutien et ses petits coups de pouces (je pense notamment au fameux carton, qui fut une petite mine d'or pour certains d'entre nous...).

Je remercie également l'équipe des neuropsychologues en particulier Alexandra Benoist, et Sandrine Wannepain, qui ont permis de mener à bien ce travail.

Je remercie toutes les personnes, patients et témoins, qui ont accepté de participer à cette étude. 
ABRÉVIATIONS.....................................................................14

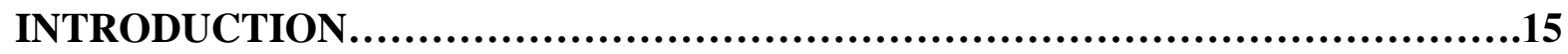
A. Généralités
B. Attention et ralentissement
C. Maladie de Parkinson et ralentissement de l'action
D. Objectifs et hypothèses

MÉTHODE
A. Population
B. Matériel
C. Analyse statistique

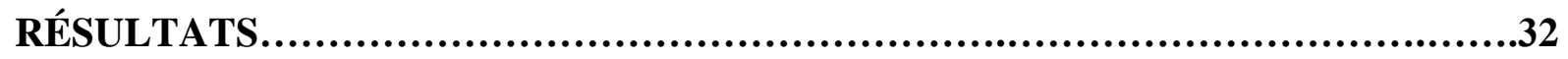

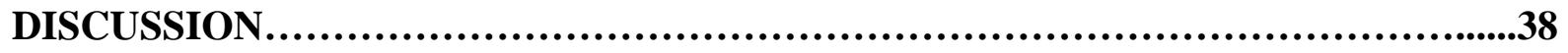

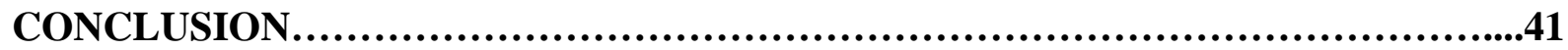

RÉFÉRENCES.....................................................................43

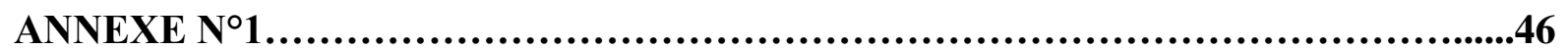

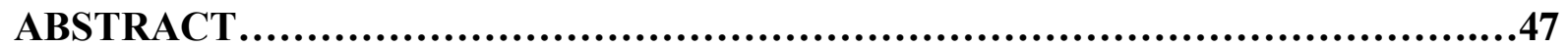

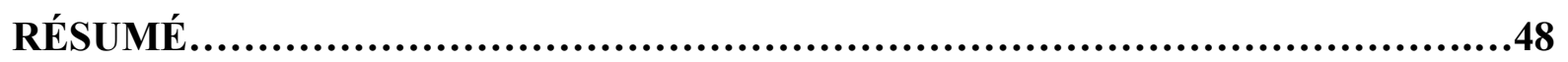




\section{ABRÉVIATIONS}

MP: Maladie de Parkinson

TR: temps de réaction

TRS: temps de réaction simple

TRC: temps de réaction à choix

FM: fréquence motrice

TIV: temps d'inspection visuel

C5: $5^{\text {ème }}$ percentile

C50: $50^{\text {ème }}$ percentile

MA: maladie d'Alzheimer

DCL: démence à corps de Lewy

UPDRS: Unified Parkinson disease rating scale

MMSE: Mini Mental Scale Evaluation 


\section{INTRODUCTION}

\section{A. Généralités}

Un ralentissement de l'action a été mis en évidence dans de nombreuses pathologies neurodégénératives telle la maladie d'Alzheimer (MA), la démence à corps de Lewy (DCL) [1], mais aussi après un accident vasculaire cérébral [2], la sclérose en plaques [3] et également dans des pathologies psychiatriques comme la dépression ou la schizophrénie . Ce ralentissement peut aussi être physiologique et survient naturellement au cours du vieillissement [4]. Il est classiquement objectivé par des tests cliniques parmi lesquels l'exploration quantitative de la marche mais également par les épreuves neuropsychologiques classiques (test de Stroop, Trail Making Test,...) et surtout par des épreuves de chronométrie mentale sous la forme de tâches de temps de réaction simple (TRS) ou temps de réaction à choix (TRC).

Ces épreuves permettent une description quantitative du ralentissement mais sont aussi capable de préciser l'origine du ralentissement de l'action. Si les mécanismes responsables de la rapidité de l'action renvoient traditionnellement à la notion d'attention, d'autres processus sont mis en jeu et peuvent contribuer à la performance des taches de temps de réaction. Selon un modèle récent proposé par Godefroy et al (2010) [4], quatre processus principaux pourraient être impliqués dans la réalisation d'un temps de réaction dont le processus attention : les processus perceptif, moteur, attentionnel et décisionnel. Sur la base de ce modèle, l'origine du ralentissement peut être différente et la variation des profils observés à partir de taches de TR pourrait contribuer en pratique clinique au diagnostic différentiel des pathologies neurodégénératives [1]. Ces éléments sont d'autant plus importants que les troubles attentionnels et le ralentissement de l'action sont observés dès les stades débutants voire prédémentiels.

Dans les pathologies des ganglions de la base, les études concernant les temps de réaction se sont focalisées sur la maladie de Parkinson (MP) [5-9]. Très peu de données existent sur les autres pathologies et les études comparatives sont très rares [10].Des résultats de Girotti et al [11], ont montré que les TRS et TRC de patients suivis pour une Paralysie Supra-nucléaire Progressive (PSP) et de patients suivis pour une maladie de Huntington (MH) étaient plus allongés que ceux de patients suivis pour une MP.

Dans la DCL, Bailon et al [1] a retrouvé un ralentissement dont le profil évoqué des troubles attentionnels et visuels qui contribuaient aux hallucinations et se différentiaient du profil 
de ralentissement observé dans la MA. En effet le profil de ralentissement observé dans la MA était évocateur d'une atteinte des processus perceptivo-moteurs, pourtant rapportés comme classiquement préservés dans cette pathologie, et des processus décisionnels.

\section{B. Attention et ralentissement}

Comme nous venons de le voir, les mécanismes responsables de la rapidité de l'action renvoient traditionnellement à la notion d'attention. Ce concept d'attention est difficile à définir, il peut toutefois se résumer par la sélection et le maintien dans la conscience d'un évènement extérieur ou d'une pensée.

Selon Posner [12], trois grandes fonctions attentionnelles (alerte, orientation, maintien et controle attentionnel) supportées par trois réseaux neuronaux peuvent être distingués. Elles constituent les trois dimensions du système attentionnel : l'intensité (Alerte); la sélectivité (Orientation); la dimension exécutive (contrôle).

La fonction d'alerte est impliquée dans l'élévation et le maintien d'un état d'alerte préparatoire à l'apparition imminente d'un stimulus. Elle recouvre l'alerte tonique qui est le niveau d'éveil cortical du sujet, il est mesuré par le temps de réaction entre la présentation d'un stimulus et sa détection (contrôle cognitif général de l'éveil), l'alerte phasique (réaction évaluée par son temps, à l'apparition d'un stimulus simple), Elle représente en fait un changement brutal de notre alerte tonique. Elle est mesurée par le même temps de réaction mais le stimulus est précédé par un signal avertisseur dont l'objectif est de prévenir le sujet afin qu'il optimise son état d'alerte. La vigilance et l'attention soutenue se définissent comme un haut niveau d'alerte sur de longs intervalles de temps (nombre de stimuli faible en situation de vigilance, élevé dans l'attention soutenue).

La fonction d'orientation recouvre l'attention sélective ou focalisée et l'attention divisée.

L'attention focalisée est la capacité qui nous permet de sélectionner une information qu'on juge pertinente ou non. Cette capacité présente 2 mécanismes importants: l'activation de l'information pertinente et l'inhibition des informations distractives. Dans ce type de paradigme, est pris en compte le temps de réaction ainsi que les erreurs lorsque le sujet répond à un stimulus non-conforme.

L'attention divisée concerne les mécanismes attentionnels qui nous permettent de réaliser simultanément 2 actions cognitives de manière efficace. Cela renvoie à des mécanismes qui permettent de gérer des productions attentionnelles. 
Quant au contrôle attentionnel, il est proche de la notion de contrôle exécutif [13], et il est impliqué dans des opérations cognitives complexes.

Les tests neuropsychologiques utilisés en clinique courante évaluant l'attention permettent de mesurer indirectement le ralentissement de l'action. En effet dans ces épreuves, la performance nécessite de réaliser un compromis rapidité précision et une mesure du temps de réalisation est couramment enregistrée. Si ces épreuves permettent d'objectiver un ralentissement, elles contiennent des opérations cognitives multiples dépassant largement le domaine de l'attention et l'interprétation de l'origine du ralentissement demeure délicate. Autrement dit, ils apparaissent intéressants dans une optique clinique mais sont difficiles à interpréter puisqu'ils reflètent les temps des différents processus mis en jeu dans la rapidité de l'action sans discernement. C'est pourquoi ont été développés des paradigmes expérimentaux de chronométrie mentale permettant d'étudier l'attention et le ralentissement de l'action à travers la mesure des temps de réaction. De plus, une analyse plus fine des processus impliqués dans une tache de réaction permettent de spécifier l'origine du ralentissement qui pourrait être observé dans ces tâches. Ainsi, comme proposé par Godefroy et al. [4], quatre processus seraient impliqués lors de la réalisation de tache de temps de réaction: les processus perceptifs, moteurs, attentionnels et décisionnels (schéma n ${ }^{\circ} \mathbf{1}$ ). Plus précisément, ce type de test requiert l'activation la plus rapide possible d'une réponse motrice préprogrammée (consistant habituellement à appuyer l'index sur un bouton, après la perception d'un stimulus prédéterminé et implique :

- Un traitement perceptif (visuel ou auditif) qui permet la détection (visuelle ou auditive) et l'identification du stimulus (temps d'identification visuelle)

- Un processus décisionnel qui permet la sélection de la réponse

- Un processus moteur qui correspond au temps nécessaire à la programmation et la réalisation motrice

- Des capacités attentionnelles afin de maintenir une optimisation des réponses tout au long de l'épreuve 


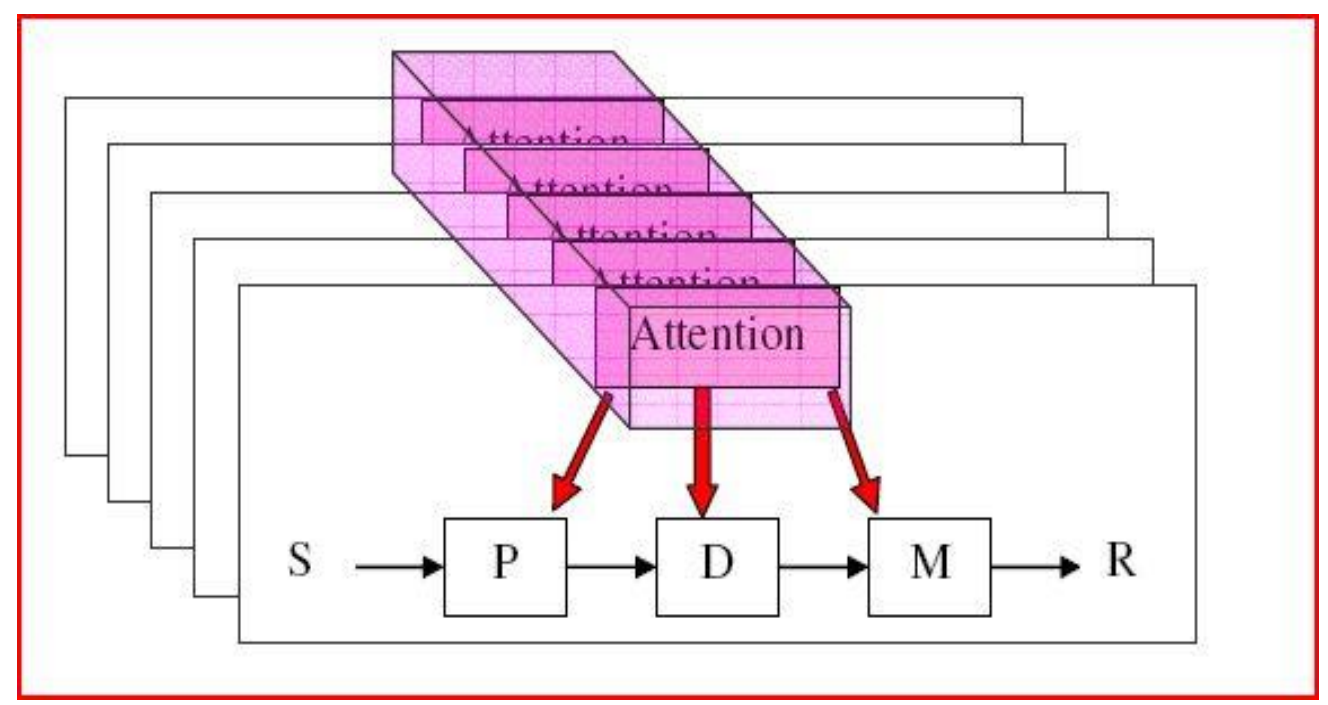

Schéma 1 : Processus impliqués dans une tâche de TR : les essais sont représentés par la superposition des plans ; chaque essai est initié par la présentation d'un stimulus $S$ et se termine par la réponse $R$. Il implique des processus perceptif $P$, décisionnel $D$ et moteur $M$. Afin d'augmenter la rapidité de la réponse, l'attention exerce une influence sur ces processus à chaque essai. Godefroy et al (2010a).

Ainsi les temps de réaction permettent une mesure quantitative fiable et reproductiblepour étudier l'attention et le ralentissement de l'action et ils permettent également de pouvoir explorer l'origine du ralentissement de l'action.

\section{Maladie de Parkinson et ralentissement de l'action}

La MP est la deuxième affection neurodégénérative après la maladie d'Alzheimer. Sa prévalence est de 2 pour 1000 dans la population générale, s'élevant à $2 \%$ chez les plus de 65 ans. Elle débute en moyenne entre 55 et 65 ans et est discrètement plus fréquente chez les hommes [14]. Elle se caractérise sur le plan anatomopathologique par une dégénérescence de la voie dopaminergique nigrostriée se traduisant cliniquement par des symptômes moteurs : akinésie, rigidité, tremblement de repos et troubles de la marche. L'akinésie se définie comme une difficulté d'initiation du mouvement, elle se mesure par un temps de réaction simple avec stimulus avertisseur. Il s'y associe un ralentissement de l'action, la bradykinésie mesurée par le temps d'exécution et une réduction de l'amplitude de l'exécution des mouvements ou hypokinésie. 
Cependant, la raréfaction neuronale atteint d'autres noyaux non dopaminergiques du tronc cérébral comme le locus cœruleus, le noyau dorsal du vague, la formation réticulée mésencéphalique, le noyau basal de Meynert rendant ainsi compte des signes non moteurs (troubles de la cognition et du comportement, troubles thymiques, troubles végétatifs, troubles du sommeil, troubles sensitifs...) qui altèrent également la qualité de vie des patients.

Les symptômes cognitifs sont initialement généralement discrets et n'interfèrent pas de façon significative avec les activités de la vie quotidienne. Le recours systématique aux tests neuropsychologiques a permis de montrer que ces changements cognitifs sont fréquents, pouvant atteindre jusqu'à $93 \%$ des patients selon l'étude de Pirozzolo et al. [15], qu'ils concernent principalement les fonctions visuo-spatiales, mnésiques et exécutives. Ces troubles peuvent être observés même au tout début de la maladie [16], et ne sont pas corrélés à la sévérité de l'atteinte motrice [17].

La mise en évidence de troubles visuo-spatiaux au cours de la maladie de Parkinson, même dans des tâches faisant peu appel à une composante motrice, a conduit certains à considérer qu'il existait un déficit visuo-spatial dans cette maladie. Cependant, on pense actuellement que les déficits observés sont directement liés à la complexité cognitive des paradigmes visuo-spatiaux qui font habituellement intervenir des capacités de rotation mentale, d'auto-élaboration de stratégies ou de planification. Ceci suggère que les troubles visuo-spatiaux observés dans ce type de tâche résulteraient plutôt d'une diminution des ressources attentionnelles ou des capacités de traitement de l'information, que d'un déficit visuo-spatial spécifique.

Plusieurs systèmes de mémoires sont altérés dans la maladie de Parkinson. Les capacités de la mémoire de travail sont diminuées, et la mémoire à long terme est également déficitaire. Le profil de déficit observé serait plutôt évocateur d'un trouble des processus stratégiques de récupération de l'information. Ainsi, les capacités de stockage qui sont sous le contrôle des structures temporales internes, seraient préservées, ainsi que le montrent les courbes d'apprentissage et de maintien d'informations après délai. En revanche, la performance des patients est diminuée dans les tâches de rappel libre qui nécessitent une organisation mentale des informations à mémoriser. C'est donc le déficit exécutif qui perturberait la performance des patients dans les tâches qui demandent d'activer spontanément des stratégies efficaces d'encodage ou de récupération.

De nombreux travaux rapportent des troubles dysexécutifs et ceci à tous les stades d'évolution de la maladie [16, 18, 19]. On entend par fonctions exécutives l'ensemble des processus mentaux nécessaires à l'élaboration de réponses cognitives ou comportementales 
adaptées à des situations nouvelles (difficultés dans la planification des actions, la mise en place de stratégies, la résolution des problèmes, le raisonnement logique, la prise de décision et la résistance à l'interférence).

Dans la MP, le dysfonctionnement induit par la déplétion dopaminergique des systèmes associatifs et des circuits reliant le striatum aux régions frontales et préfrontales est souvent considéré comme la principale cause du déficit attentionnel et du syndrome dysexécutif [20]. Les troubles exécutifs les plus fréquents sont les troubles de la flexibilité mentale.

Quant au ralentissement, de nombreux travaux aux résultats contradictoires ont étudiés le ralentissement de l'action et les troubles attentionnels dans la maladie de Parkinson. Certains de ces travaux ont ainsi tentés d'identifier l'origine mais celle-ci reste malgré tout indéterminée [2123]. A ce jour la comparaison des TRC entre parkinsoniens et témoins a retrouvé des résultats discordants et controversés quelque soit le paradigme de temps de réaction employé. Ainsi, Jordan et al [24] retrouvent un allongement significatif pour les TRS (tâche dans laquelle le patient doit répondre toujours à un même stimulus) des patients parkinsoniens par rapport aux témoins alors qu'aucune différence significative n'est retrouvée pour le TRC (tache dans laquelle le patient doit répondre à des stimuli différents). Aussi la latence décisionnelle qui est la différence entre le TRC et le TRS apparaît dans certaine étude plus courte chez les sujets parkinsoniens que chez les témoins [25]. L'interprétation des auteurs est que les sujets sains peuvent dans les TRS anticiper la réponse puisqu'il s'agit toujours du même mouvement alors que cela n'est plus possible pour les TRC puisque la réponse varie, alors que les sujets parkinsoniens ont perdu cette capacité de pré-programmation dans les TRS.

De nombreuses études se sont également intéressées aux effets du traitement dopaminergique sur le ralentissement avec des résultats discordants [8, 26-28]. La plupart des études ne retrouvent pas de différence entre les performances en condition On et Off. Rafal et al. [27] ont étudié en TRS, quatre patients avant et après instauration du traitement dopaminergique initial et dix patients en condition On et Off et ont ainsi montré que les TRS étaient plus lents chez les patients avant instauration du traitement dopaminergique et ceux en condition $O f f$. Au contraire, Pulmann et al.[8], retrouvent une amélioration des TRC en condition On mais pas des TRS, tandis que Starkstein et al. [29] ne retrouvent aucune modification quelque soit la condition en TRS.

Il est admis dans la MP l'existence de déficits sensoriels et notamment des difficultés d'intégration des informations visuo-spatiales : anomalies de la perception du mouvement, du contraste des couleurs, de la profondeur et de la coordination visuo-motrice [30, 31]. Plusieurs études [32-34] ont ainsi mis en évidence un allongement du temps d'inspection visuel (TIV) et 
cela indépendamment de l'atteinte motrice puisqu'il n'y avait pas de différence significative entre les tests réalisés en condition $O n$ et $O f f$ suggérant qu'une altération des processus perceptifs visuels pourrait participer au ralentissement de l'action dans la MP. Dans l'étude de Johnson (32), seule la mesure du temps d'identification visuelle était retenue en faisant varier le temps d'exposition du stimulus (500ms, 250ms...), permettant de confirmer un ralentissement présent dès la phase d'identification visuelle. Cependant, d'autres études portant sur les TIV n'ont pas mis en évidence leur allongement [35].

Certaine études se sont intéressées à l'attention divisée montrant à partir d'un paradigme de double tâche, un déficit attentionnel. Malapani et al [36] ont montré que dans la condition où les patients parkinsoniens devaient effectuer deux épreuves de TR à choix (visuelle ou auditive) de façon séparée ou simultanée, les patients parkinsoniens en condition Off avaient des performances normales pour chacune des deux épreuves effectuées séparément, et présentaient un allongement anormal quand les tâches devaient être réalisées simultanément. Cela suggère que les patients parkinsoniens éprouvent des difficultés à partager leurs ressources attentionnelles. Toutefois dans ce domaine également de nombreuses incertitudes demeurent, puisque d'autres études ne rapportent pas de déficit d'attention divisée dans la MP [37-39].

En résumé l'ensemble de ces travaux montrent des incertitudes quant au ralentissement et aux troubles attentionnels dans la maladie de Parkinson avec des résultats contradictoires entre les différentes études. Plusieurs raisons peuvent être évoquées dont principalement l'absence de définition précise du ralentissement de l'action dans la MP et l'absence d'outil standardisé et validé permettant d'explorer le ralentissement, et aussi l'inclusion de population de patients hétérogènes. En effet, ces études ont été réalisées sur des populations différentes avec des indices de mesure de temps de réaction différents puisqu'il n'existait pas jusqu'à présent de paradigme standardisé. Ce qui est par contre admis par tous est que le ralentissement de l'action dans la MP ne résulte pas uniquement d'un ralentissement moteur mais également de facteurs cognitifs et attentionnels. 


\section{Objectifs et hypothèses}

Dans ce contexte, l'objectif principal de ce travail était d'objectiver le ralentissement de l'action chez des patients ayant une maladie de Parkinson au moyen d'une batterie des tests de chronométrie mentale informatisée validée et standardisée dans des conditions d'évaluation $O n$ et $O f f$ [4].

Les objectifs secondaires étaient d'explorer au moyen de cette même batterie le mécanisme du ralentissement de l'action, à savoir si ce ralentissement est d'origine perceptif, moteur, décisionnel ou attentionnel.

\section{MÉTHODE}

\section{A. Population}

Nous avons inclus de manière prospective entre juillet 2011 et mars 2014, 40 patients atteints de la MP définie par les critères de l'United Kingdom Parkinson's disease Society Brain Bank (UKPDSBB) selon les critères d'inclusion suivants : (1) langue maternelle française ; (2) stades 1 à 3 de Hoehn et Yahr [40]. Nous avons exclu les patients qui présentaient : (1) des difficultés d'écriture ou de lecture; (2) un déficit perceptif visuel ou auditif empêchant la lecture, le dessin, l'écriture ou la compréhension des consignes ; (3) des hallucinations visuelles ; (4) un syndrome démentiel défini selon les critères du DSM IV; (5) des antécédents significatifs pouvant retentir sur la cognition (dysthyroïdie non équilibrée, cardiopathie ischémique ou emboligène non stabilisée ou symptomatique); (6) des antécédents neurologiques actuels ou passés (Accident vasculaires hémorragiques ou ischémiques, traumatisme crâniens, épilepsie, ...) ; (7) des pathologies psychiatriques sauf dépression traitée sous réserve d'un traitement stable depuis 1 mois; (8) un traitement psychotrope (sauf anxiolytique ou traitement antidépresseur stable depuis 1 mois).

Dans le même temps, 40 témoins sains ont participé à l'étude. Ils étaient appariés selon l'âge et le nombre d'années de scolarité avec les patients parkinsoniens. Ils ont été recrutés dans l'entourage des examinateurs ou des patients. Un entretien a permis de recueillir les données démographiques, les antécédents médico-chirurgicaux, les traitements médicamenteux et le score 
au MMSE. Les critères d'exclusion des témoins étaient les mêmes que les patients avec un score au MMSE devant se situer dans les normes [41].

\section{Caractéristiques démographiques}

Pour chacun des patients et témoins des données démographiques ont été recueillis âge, sexe, la latéralité manuelle, nombre d'années de scolarité, le niveau scolaire (1 : pas de diplôme ou certificat d'études primaires (CEP); 2 : certificat d'aptitudes professionnelles (CAP), brevet d'enseignement professionnel (BEP) ; 3 : BAC réussi ou plus,).

Les caractéristiques démographiques des deux groupes sont présentées dans le tableau $\mathrm{n}^{\circ} 1$ cidessous.

\begin{tabular}{|c|c|c|c|}
\hline & $\begin{array}{l}\text { MP } \\
(n=40)\end{array}$ & $\begin{array}{l}\text { Témoins } \\
(n=40)\end{array}$ & $\mathbf{p}$ \\
\hline Age (années) & $66.5 \pm 7.7$ & $64.5 \pm 9.0$ & 0.30 \\
\hline Sexe H/F & $26 / 14$ & $20 / 20$ & 0.26 \\
\hline Latéralité D/G & $39 / 1$ & $39 / 1$ & 0.00 \\
\hline Nombre d'années d'étude & $11.2 \pm 3.1$ & $11.7 \pm 3.4$ & 0.59 \\
\hline Niveau d'éducation & 1.9 & 1.9 & 0.90 \\
\hline Empan & 5 & 5 & 1 \\
\hline MMSE (/30) & $26.7 \pm 2.2$ & $29.5 \pm 0.8$ & 0.0001 \\
\hline
\end{tabular}

Tableau $\mathrm{n}^{\circ} 1$ : Comparaison des caractéristiques démographiques des patients parkinsoniens et des témoins. Les données sont exprimées en moyenne \pm écart-type. La comparaison de groupe a été effectuée à l'aide du test $t$. Significativité $\mathrm{p}<0,05$.

\section{Caractéristiques cliniques}

Pour chacun des patients des données cliniques ont été recueillis :

(1) âge de début de la maladie, durée d'évolution.

(2) antécédents significatifs : neurologiques, cardiologiques et psychiatriques.

(3) traitements dopaminergiques et autres. 
Un examen clinique a été réalisé comprenant les parties II, III et IV de la Unified Parkinson's Disease Rating Scale (UPDRS; The Unified Parkinson's Disease Rating Scale [42]) en conditions $O n$ et $O f f$.

L'acuité visuelle était mesurée par l'échelle de Parinaud binoculaire et l'acuité auditive était évaluée de manière subjective lors de la consultation.

Les principales caractéristiques cliniques des sujets parkinsoniens sont présentées dans le tableau $\mathbf{n}^{\circ} \mathbf{2}$ ci-dessous.

\section{SYMPTOMES PARKINSONIENS}

\begin{tabular}{|l|l|}
\hline Age de début & $59.0 \pm 9.6$ \\
\hline Durée d'évolution & $7.8 \pm 3.9$ \\
\hline Stade Hoehn et Yahr & $2.2 \pm 0.4$ \\
\hline UPDRS II en ON (/52) & $10.8 \pm 5.3$ \\
\hline Score UPDRS III moteur OFF (/108) & $27.3 \pm 9.5$ \\
\hline Sous score bradykinésie OFF ( /36) & $11.1 \pm 4.0$ \\
\hline Score UPDRS III moteur ON (/108) & $14.3 \pm 6.1$ \\
\hline Sous-score bradykinésie ON (/36) & $6.1 \pm 3.4$ \\
\hline Parinaud & $2.8 \pm 0.9$ \\
\hline TRAITEMENTS & \\
\hline $\begin{array}{l}\text { Traitement dopaminergique en } \text { mg équivalent L-dopa } \\
\text { par jour (mg/j) (voir annexe n'1) }\end{array}$ & $958 \pm 605$ \\
\hline \begin{tabular}{l} 
Agoniste dopaminergique n(\%) \\
\hline L-dopa n(\%)
\end{tabular} & 47.5 \\
\hline
\end{tabular}

Tableau $\mathbf{n}^{\circ} 2$ : Données cliniques et thérapeutiques des patients parkinsoniens. Données cliniques exprimées en moyenne \pm écart-type, données thérapeutique exprimées en pourcentage de patients prenant les traitements.

Les patients ont été sélectionnés en consultation par le neurologue au cours de leur suivi au CHU d'Amiens. L'étude a concerné tous les patients acceptant de participer à l'étude et pour lesquels une courte hospitalisation en hôpital de semaine de Neurologie pour la réalisation d'examens nécessaires à leur suivi était prévue (évaluations clinique, neuropsychologique, test d'administration aigue de L-dopa et tout autre examen complémentaire). Ce bilan a été complété par la passation des tests chronométriques de temps de réaction. Nous avons profité de la réalisation d'administration aigüe de L-Dopa pour évaluer les patients en temps de réaction dans un premier temps en condition $O f f$ soit à jeun de tout traitement dopaminergique depuis la veille 
à minuit, puis dans un second temps en condition $O n$ soit 1 heures 30 après la prise d'une dose supraliminaire de Modopar dispersible ${ }^{\circledR}$ et la reprise du traitement habituel.

Tous les sujets inclus, patients et témoins ont bénéficié d'une information claire, loyale et intelligible sur le protocole de recherche. Les témoins ont signé un consentement éclairé et tous ont lu une lettre d'information. Le protocole a été promu par le CHU d'Amiens et a reçu l'approbation du Comité de Protection des Personnes Nord Ouest II.

\section{Caractéristiques neuropsychologiques}

Le bilan neuropsychologique a été réalisé une fois, en condition «Simple-On » soit avec prise du traitement habituel, la durée de passation était d'environ 1h30. Il s'agissait d'un bilan standard réalisé en pratique courante nécessaire à l'évaluation neuropsychologique de tout patient parkinsonien [43].

Le bilan neuropsychologique comprenait une évaluation de l'efficience intellectuelle générale, du langage, des capacités d'exploration visuo-spatiale, des capacités visuo-constructives, des capacités mnésiques et des fonctions exécutives. Plus spécifiquement, le bilan évaluait :

- L'efficience cognitive globale grâce au MMSE [44], de la Mattis Dementia Rating Scale (MDRS, [45]).

- Le langage par le Token test abrégé si problème de compréhension verbale orale [46], le test de dénomination orale « DO $80 »[47]$ et les tests de fluences verbales [48].[49]

- Les capacités d'explorations visuo-spatiales par le test de barrage d'Albert [49], et les gnosies visuelles à partir du protocole d'évaluation des gnosies visuelles (PEGV) [50]. Les capacités visuo-constructives à partir de la figure de Rey [51]

- Les capacités mnésiques à court terme à partir du test d'empan et à long terme épisodique à partir du test des portes, RL/RI16 items [52, 53]

- Les fonctions exécutives à partir de la batterie du GREFEX (2008) : Trail Making Test (TMT) [54], test de classement de cartes du Wiscontin modifié (Modified card sorting test, MCST ; [55], test de Brixton (Burgess [56], le test de Stroop [57]

Les troubles de l'humeur (dépression, et anxiété étaient également évalués à partir d'autoquestionnaires. (échelle de Montgomery Asberg Depression Rating Scale (MADRS), [58] ; échelle de Golberg). 
.Les performances moyennes obtenues par les patients parkinsoniens aux différents tests sont présentés dans le tableau $\mathbf{n}^{\circ} \mathbf{3}$.

\begin{tabular}{|c|c|c|}
\hline & Performances & $\begin{array}{l}\% \text { de sujets } \\
\text { déficitaires }\end{array}$ \\
\hline MMSE (/30) & $26.7 \pm 2.2$ & $0(0 / 40)$ \\
\hline DRS (/144) & $135.6 \pm 5.5$ & $30.6(11 / 36)$ \\
\hline PM47 & $28.3 \pm 4.3$ & $0(0 / 28)$ \\
\hline MADRS & $7.6 \pm 5.9$ & $50(16 / 32)$ \\
\hline 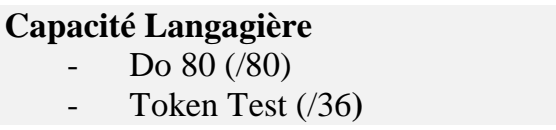 & $\begin{array}{l}78.6 \pm 1.6 \\
33.2 \pm 1.8\end{array}$ & $\begin{array}{l}2.8(1 / 36) \\
0(0 / 21)\end{array}$ \\
\hline $\begin{array}{cl}\text { Exploration VS et capacités VC : } \\
-\quad \text { Albert (omissions) } \\
-\quad \text { Figure de Rey }(/ 36)\end{array}$ & $\begin{array}{l}0.3 \pm 0.6 \\
30.2 \pm 4.4\end{array}$ & $\begin{array}{l}0(0 / 36) \\
10.3(3 / 29)\end{array}$ \\
\hline $\begin{array}{l}\text { Mémoire à court-terme : } \\
-\quad \text { Empan endroit }\end{array}$ & $5.4 \pm 1.2$ & $11.8(4 / 34)$ \\
\hline Mémoire épisodique visuelle (/24) & $16 \pm 3.8$ & $14.3(5 / 35)$ \\
\hline \multicolumn{3}{|l|}{ Mémoire à long terme: } \\
\hline RIM & $14.7 \pm 1.5$ & $16.7(6 / 36)$ \\
\hline RL3 & $11 \pm 2.9$ & $16.7(6 / 36)$ \\
\hline RT3 & $16 \pm 1.3$ & $8.3(3 / 36)$ \\
\hline Reconnaissance & $16 \pm 0.6$ & $8.3(3 / 36)$ \\
\hline RLD & $11 \pm 2.7$ & $16.7(6 / 36)$ \\
\hline RTD & $16 \pm 1.5$ & $8.3(3 / 36)$ \\
\hline \multicolumn{3}{|l|}{ Capacités perceptives PEGV : } \\
\hline - $\quad$ Figures identiques & $9.6 \pm 0.6$ & $0(0 / 34)$ \\
\hline - $\quad$ Figures enchevêtrées & $33.4 \pm 2.8$ & $8.8(3 / 34)$ \\
\hline - Appariement fonctionnel & $10 \pm 0$ & $0(0 / 32)$ \\
\hline - Appariement catégoriel & $9.9 \pm 0.4$ & $6.3(2 / 32)$ \\
\hline \multicolumn{3}{|l|}{ Fonctions exécutives : } \\
\hline TMT - Index temps & $114.9 \pm 81.9$ & $15.2(5 / 33)$ \\
\hline - Erreurs Alt & $0.2 \pm 0.4$ & $0(0 / 33)$ \\
\hline Stroop Index interférence - Temps & $74.6 \pm 59$ & $2.94(1 / 34)$ \\
\hline - Erreurs & $1.1 \pm 1.7$ & $15.6(5 / 32)$ \\
\hline Brixton & $22 \pm 8.61$ & $12.5(4 / 32)$ \\
\hline Fluences littérales $\mathrm{Nb}$ mots émis & $15.4 \pm 5.9$ & $3(1 / 33)$ \\
\hline Fluences catégorielles $\mathrm{Nb}$ mots émis & $23 \pm 6.8$ & $17.6(6 / 34)$ \\
\hline MCST : - Nb catégories & $4.8 \pm 1.7$ & $12.1(4 / 33)$ \\
\hline - Erreurs & $10.5 \pm 9.8$ & $9.1(3 / 33)$ \\
\hline - Erreurs P & $3.2 \pm 3.6$ & $6.1(2 / 33)$ \\
\hline Tâche double Mu & $91.62 \pm 12.32$ & $3.2(1 / 31)$ \\
\hline
\end{tabular}

Tableau $\mathbf{n}^{\circ} 3$ : Données neuropsychologiques. RIM : rappel immédiat, RL : rappel libre, RT : rappel total, $\mathrm{RD}$ : rappel différé. $\%=$ Pourcentage d'altération; VS $=$ Visuo-Spatiales ; VC $=$ Visuo-Constructives ; PEGV : Protocole d'évaluation des gnosies visuelles ; TMT : Trail making test. 
Une analyse individuelle des performances a été réalisée avec un score seuil selon les normes publiées. Les résultats de cette analyse montraient que tous les patients avaient des capacités langagières (compréhension orale) et d'exploration visuo-spatiale préservées. En ce qui concerne la mémoire épisodique verbale, 17\% des patients, soit 6 patients sur 36 avaient un déficit ; quatre de ces six patients avait un profil en faveur d'un trouble de la récupération selon les critères proposés par Godefroy et al [59].Concernant les fonctions instrumentales, seule une altération des capacités visuo-constructives était constatée chez 10\% des patients soit 3 patients sur 29.

En résumé le profil neuropsychologique des patients inclus dans cette étude est proche de celui retrouvé ds la littérature avec une relative préservation de l'efficience cognitive globale alors qu'il existe une atteinte des capacités visuo-constructives et de la mémoire épisodique verbale dont le profil est en faveur d'un trouble de la récupération et une atteinte des fonctions exécutive en particulier un trouble de la flexibilité mentale et un trouble de la génération d'information.

\section{B. Matériel}

Les tests chronométriques ont été administrés sur un PC portable muni d'un écran 15 pouces. Après vérification de l'acuité visuelle. Le sujet était assis à environ $50 \mathrm{~cm}$ de l'écran. La réponse motrice était mesurée par la dépression par le doigt de la main dominante d'un bouton réponse relié à l'ordinateur. Chez le patient parkinsonien, ces tests ont été réalisés dans deux conditions : en condition Off (à jeun de tout traitement dopaminergique depuis la veille minuit), et en condition On (1h30 après la prise d'une dose supraliminaire de L-Dopa). Chez les témoins, une seule passation était réalisée.

Le protocole validé et standardisé [4] était composé de quatre tests dont la passation était réalisée dans un ordre fixé : le temps d'inspection visuel (TIV), le temps de réaction simple (TRS) réalisée en condition simple et duelle, la fréquence motrice $(\mathrm{FM})$ et le temps de réaction à choix (TRC). Ces épreuves sont détaillées dans les paragraphes ci-dessous. La condition duelle consistait pour le sujet à réaliser simultanément la tache de TRS et une tache de rappel sériel à l'empan. Cette dernière consistait pour le patient à répéter des séries de chiffres à la longueur de son empan.

Les performances obtenues (TIV, TRS en condition simple et duelle, TRC et fréquence motrice) étaient exportés vers une base de données Excel. 
Cette tâche mesurait la rapidité de perception des principales caractéristiques physiques d'un stimulus. Un signal avertisseur apparaissait au centre de l'écran blanc puis était remplacé par une figure géométrique qui se composait de deux lignes verticales reliées en leur sommet par une ligne horizontale. L'une des deux lignes était de manière aléatoire plus courte que l'autre puis un masque apparaissait sur l'écran (voir schéma n ${ }^{\circ}$ ).

La tâche consistait à identifier le côté où la ligne était la plus courte (droit ou gauche) et d'appuyer en fonction sur le bouton réponse droit ou gauche du côté de la main dominante.

Deux sessions d'entraînement étaient réalisées au décours desquelles le patient devait obtenir $100 \%$ de bonnes réponses (temps de présentation du stimulus $500 \mathrm{~ms}$ puis $200 \mathrm{~ms}$ ). Puis lors de la phase de test, un algorithme était utilisé permettant de diminuer le temps de présentation du stimulus en fonction des bonnes réponses du sujet permettant ainsi de déterminer la durée minimale nécessaire pour discriminer correctement $80 \%$ des stimuli.
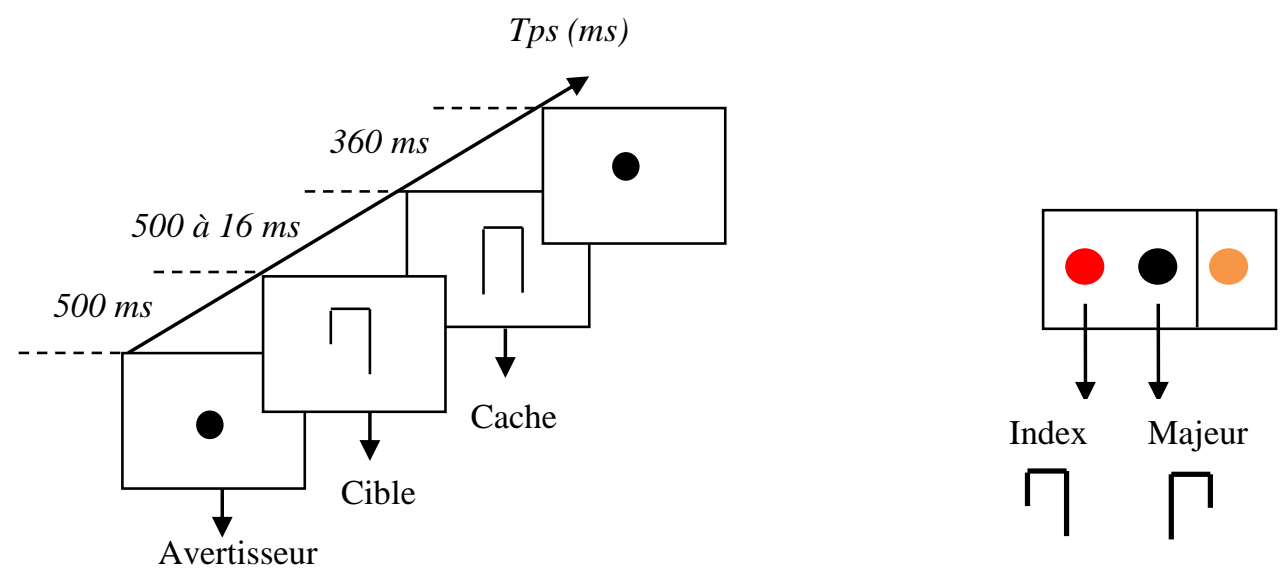

Schéma 2: Epreuve d'Inspection Visuelle.

Le temps de réaction simple (TRS) [60] :

Un stimulus avertisseur apparaissait au centre de l'écran blanc pendant $300 \mathrm{~ms}$ (croix noire). A la disparition du stimulus avertisseur, un stimulus cible apparaissait sous la forme d'une lettre noire majuscule : C, H, S ou T au centre de l'écran avec un intervalle inter-stimuli variant de manière aléatoire $(350,400,450,500,550$ et 600ms), afin de limiter les phénomènes 
d'anticipation. A l'apparition du stimulus cible, le sujet devait appuyer sur le bouton réponse et le temps de réaction (TR) était alors enregistré automatiquement. Cette épreuve était composée de 100 stimuli cibles et était effectué deux fois de suite, les mesures étant réalisées sur le second test. La performance était évaluée par le TR moyen (en ms), le nombre de bonnes réponses, d'anticipation et d'omissions.

L'épreuve était réalisée dans un premier temps en simple tâche puis dans un second temps en condition de double tâche. Condition duelle au cours de laquelle le sujet devait effectuer simultanément le test de TRS et une tache de rappel sériel à l'empan ; Cette tache consistait à répéter des séries de chiffres correspondant à l'empan du sujet avant l'épreuve.

Les TRS < $100 \mathrm{msec}$ ont été considérés comme des anticipations et les TRS > $1500 \mathrm{msec}$ comme des omissions.
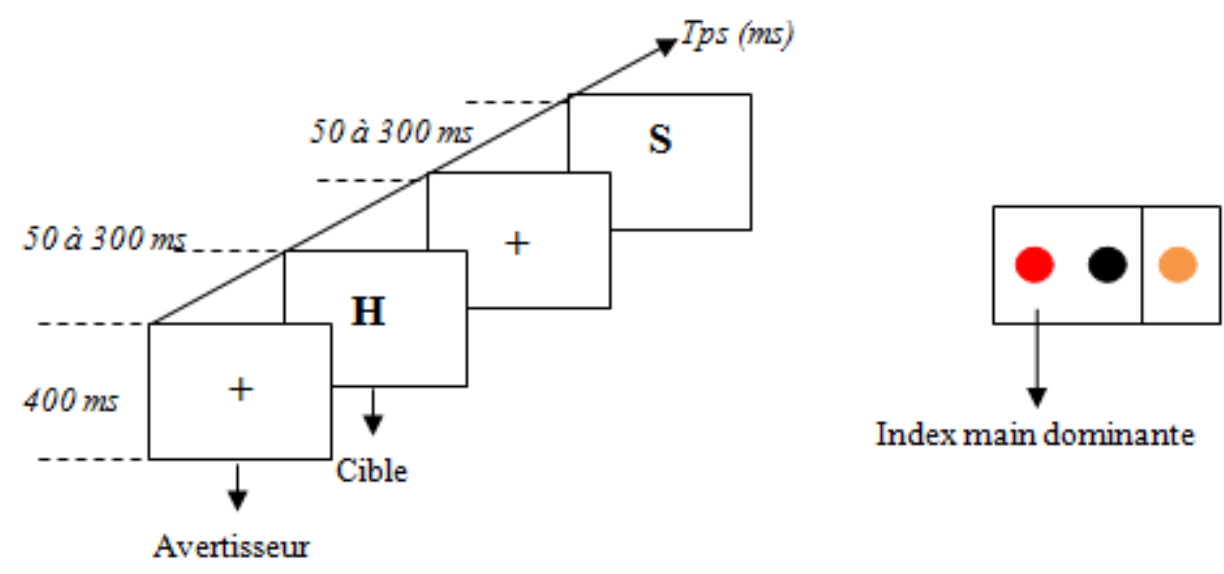

Schéma 3 : Epreuve de Temps de Réaction Simple.

La fréquence motrice (FM) ou tapping digital :

Les sujets devaient effectuer des appuis répétés le plus rapidement possible sur le bouton réponse avec l'index de la main dominante pendant 15 secondes sans lever le poignet. Dans un premier temps, l'injonction « prêt? » apparaissait au centre de l'écran pendant $100 \mathrm{~ms}$ puis vient le mot «partez ! » qui signalait aux patient de commencer les appuis répétés. La fin du test était 
signalée par le terme «c'est fini » qui s'inscrivait au milieu de l'écran. L'indice de performance retenue était la réciproque de la fréquence motrice exprimée en ms (1000ms/ Fréquence motrice).
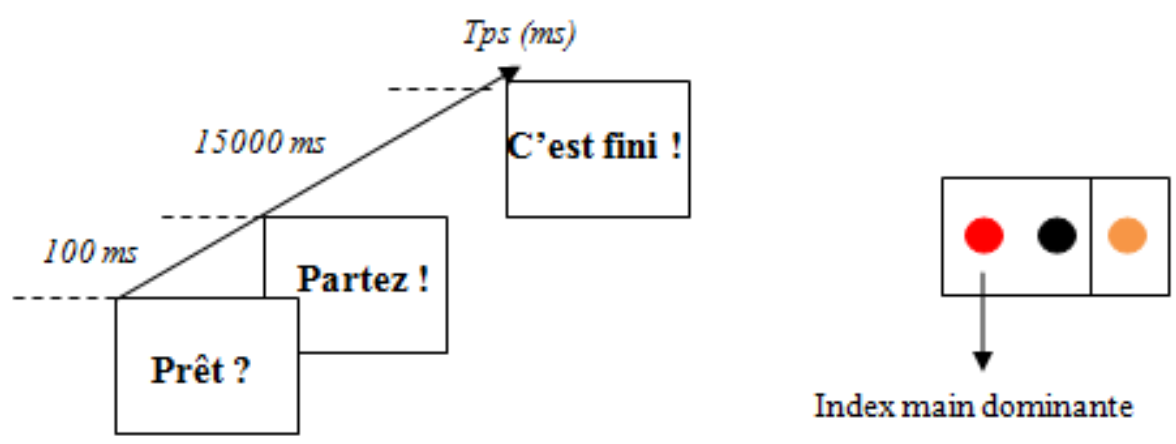

Schéma 4 : Epreuve de Fréquence Motrice.

Le temps de réaction à choix (TRC) :

Cette épreuve utilisait les mêmes stimuli que les TRS cependant les modalités de réponses différaient selon la lettre présentée : les lettres $\mathrm{C}$ et $\mathrm{S}$ étaient associées à un premier bouton réponse (index) et les lettres $\mathrm{H}$ et $\mathrm{T}$ à un deuxième bouton réponse (majeur). Chaque lettre était présentée de manière équiprobable. Cette tâche était réalisée à deux reprises, les mesures étaient effectuées sur le deuxième test. La performance était évaluée par le TR moyen (en ms), les nombres de bonnes réponses, omissions et anticipations. La latence décisionnelle correspondant à la soustraction du TRS moyen au TRC moyen a ainsi pu être calculée [61]. 

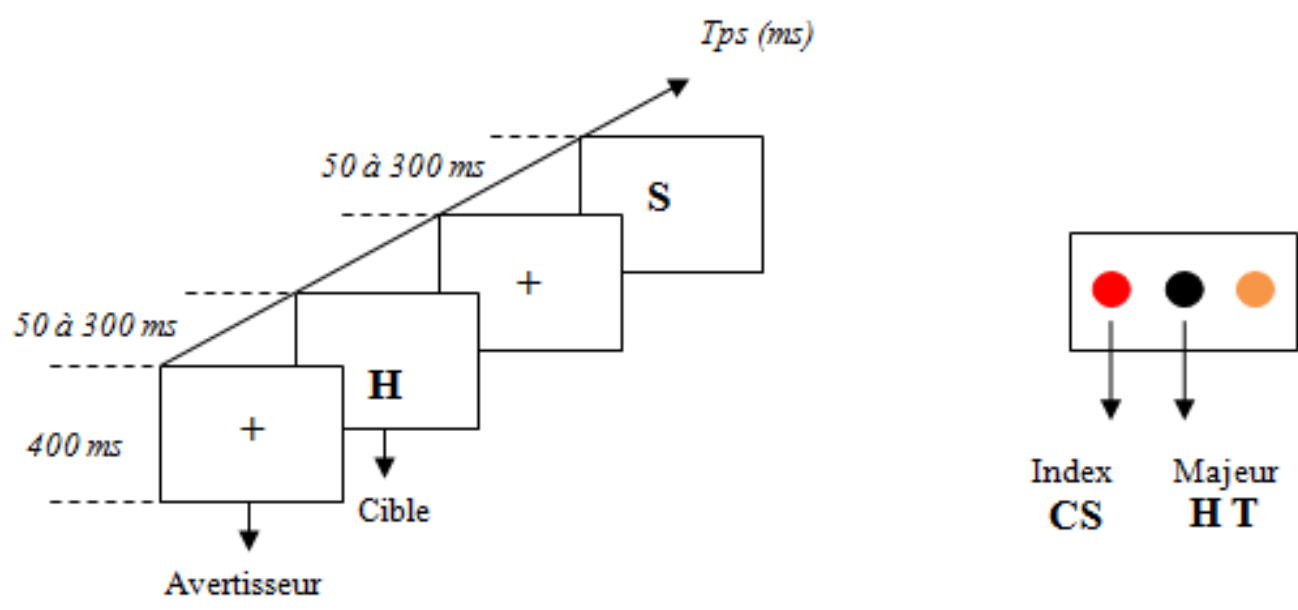

Schéma 5 : Epreuve de Temps de Réaction à Choix.

\section{Analyses statistiques}

Les analyses statistiques ont été réalisées à l'aide du logiciel SPSS (Statistical Package for the Social Sciences) et le seuil de significativité choisi était $\mathrm{p}<0,05$, sauf indiqué différemment.

La plupart des analyses consistaient en des analyses de comparaisons de groupe réalisées à l'aide de tests $t$ de Student ou d'analyse de variance multivariées (MANOVA) avec mesures répétées avec le groupe (MP, témoin) comme facteur intergroupe et les conditions expérimentales comme facteur intragroupe. Lorsque les critères de normalité des distributions n'étaient pas respectés, de tests non paramétriques ont été utilisés. Les comparaisons a postériori étaient réalisées au moyen du test de Mann-Whitney pour les groupes indépendants. Les analyses ont été conduites après transformations logarithmiques. 


\section{RÉSULTATS}

\section{Temps d'inspection visuelle}

Les performances moyennes de temps de présentation des stimuli exprimées en millisecondes pour lesquels les sujets obtiennent au minimum $80 \%$ de réponses correctes sont données dans le tableau $\mathbf{n}^{\circ} \mathbf{4}$.

\begin{tabular}{|l|l|l|}
\hline & MP & Témoins \\
\hline TIV (ms) On & $182.1 \pm 187.1$ & $114.7 \pm 98.9$ \\
\hline TIV (ms) Off & $172.2 \pm 189.5$ & $114.7 \pm 98.9$ \\
\hline
\end{tabular}

Tableau $\mathbf{n}^{\circ} 4$ : Données des temps d'inspection visuelle

Résultats exprimés en moyenne \pm écart-type. MP : Maladie de Parkinson, TIV : temps d'inspection visuelle, ms : millisecondes.

Deux analyses de comparaison ont été réalisées, une sur le groupe (MP, témoins), une sur la condition $O n$ et $O f f$. Les résultats montraient un allongement significatif du TIV des sujets parkinsoniens par rapport aux témoins $(\mathrm{t}=2,470 ; \mathrm{p}=0,02)$. L'analyse de comparaison des parkinsoniens en condition $O n$ et $O f f$ n'a pas mis en évidence de différence significative entre les deux conditions $(\mathrm{t}=0,830 ; \mathrm{p}=0,41)$.

Au total, ces analyses montraient un allongement significatif du TIV des sujets parkinsoniens en condition On par rapport aux témoins alors qu'aucune différence significative entre les la condition Off et la condition On n'a été retrouvée.

\section{Temps de réaction simple}

Les TRS moyens aux centiles C5, et C50 en simple tâche pour chaque groupe de sujet sont présentés dans le tableau $\mathbf{n}^{\circ} \mathbf{5}$ et le graphique $\mathbf{n}^{\circ} \mathbf{1}$. Pour les sujets parkinsoniens les résultats des deux conditions $O n$ et $O f f$ sont présentés. 


\begin{tabular}{|c|c|c|c|c|}
\hline & & Condition & MP & Témoins \\
\hline \multirow{4}{*}{$\begin{array}{l}\text { TRS } \\
(\mathrm{ms})\end{array}$} & C5 simple & On & $211.1 \pm 72$ & $225 \pm 69$ \\
\hline & C5 simple & Off & $248 \pm 91$ & $225 \pm 69$ \\
\hline & C50 simple & On & $340 \pm 109$ & $318 \pm 79$ \\
\hline & C50 simple & Off & $372 \pm 122$ & $318 \pm 79$ \\
\hline
\end{tabular}

Tableau $n^{\circ} 5$ : Données des temps de réaction simple

Résultats exprimés en moyenne \pm écart-type. MP : Maladie de Parkinson, C5 : $5^{\mathrm{e}}$ percentile, C50 : $50^{\mathrm{e}}$ percentile, $\mathrm{ms}$ : millisecondes

\section{- $\quad$ Performances globales aux TRS moyens}

La comparaison entre patients en condition $O n$ et témoins sur les TRS moyens fut examinée par une MANOVA avec mesures répétées sur les facteurs intra-groupe : la condition (simple, double) et les centiles (TRSC5, TRSC50); et intergroupe : le facteur groupe (MP, témoins).

L'analyse montra :

(1) une absence d'effet du groupe $\mathrm{F}(1)=0,04 ; \mathrm{p}=0,85$,

(2) un effet du centile $\mathrm{F}(1,78)=516 ; \mathrm{p}=0,0001$, lié à un TRSC50 plus long que le TRSC5 $\mathrm{p}=0,0001$

(3) Un effet de la condition, lié à un ralentissement plus important dans la condition double, mais sans différence significative entre le groupe MP et le groupe témoin.

(4) une interaction groupexcentile $\mathrm{F}(1,78)=10,8 ; \mathrm{p}=0.002$ lié à un TRSC5 légèrement plus court chez les sujets parkinsoniens $\mathrm{p}=0,002$ et un TRSC50 légèrement plus court chez les sujets témoins $\mathrm{p}=0.002$. $\left(\right.$ cf graphique $\left.\mathrm{n}^{\circ} 1\right)$

L'analyse du TRS médian a utilisé un test t par série appariée comparant les parkinsoniens en condition $O n$ et $O f f$ et a mis en évidence un allongement significatif du TRS médian en Off par rapport à la condition $O n, \mathrm{t}=2.48 ; \mathrm{p}=0.02$. 


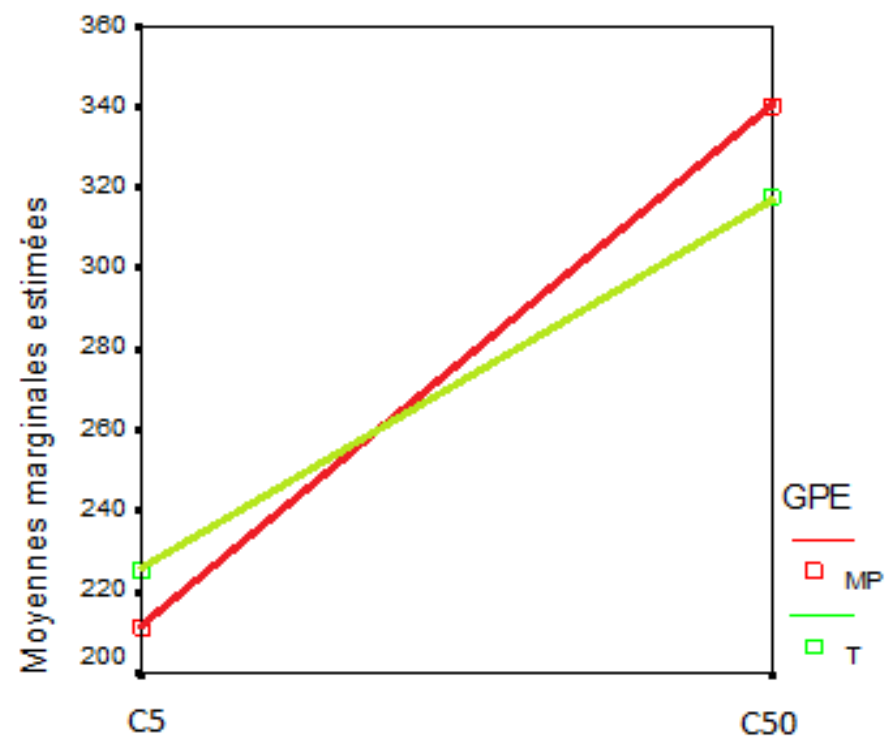

Graphique $\mathrm{n}^{\circ} 1$ : Temps de réaction simple (msec) correspondant au $5^{\text {ème }}$ centile (C5), 50 ${ }^{\text {ème }}$ centile (C50) ou TRS médian. Comparaison des témoins et des MP en condition $\mathrm{On}$.

Au total, ces analyses montraient une absence de ralentissement des TRS des parkinsoniens en condition On par rapport aux témoins, et un ralentissement significatif des TRS médians des parkinsoniens en condition Off par rapport à la condition On.

- $\quad$ Analyse des taux d'omission et d'anticipation

Les nombres d'omissions et d'anticipations pour les TRS simple en condition On et $O f f$ sont présentés dans le tableau $\mathbf{n}^{\circ} \mathbf{6}$.

\begin{tabular}{|c|c|c|c|c|}
\hline & & Condition & MP & Témoins \\
\hline \multirow[b]{2}{*}{$\begin{array}{l}\text { Omission } \\
\text { (nombre) }\end{array}$} & Simple & On & $0,6 \pm 1,5$ & 0 \\
\hline & Simple & Off & $0,1 \pm 0,3$ & 0 \\
\hline \multirow[b]{2}{*}{$\begin{array}{l}\text { Anticipation } \\
\text { (nombre) }\end{array}$} & Simple & On & $17,4 \pm 20,0$ & $10,0 \pm 1,7$ \\
\hline & Simple & Off & $11,2 \pm 17,5$ & $10,0 \pm 1,7$ \\
\hline
\end{tabular}

Tableau $n^{\circ} 6$ : Données des anticipations et omissions. 
Une analyse de variance sur le taux d'omissions et le taux d'anticipation avec comme facteur inter-sujet le facteur groupe (MP, Témoins), et intra groupe la condition $O n$ et $O f f$ a été utilisé. Les résultats de l'analyse montraient un effet du groupe sur le taux d'omission et d'anticipation $(\mathrm{F}(1)=4.45 ; \mathrm{p}=0.04)$, lié à un taux d'omission et d'anticipation plus important chez les sujets MP par rapport aux témoins.

Puis un test t par série appariée a comparé les parkinsoniens en condition On et Off et a mis en évidence une différence significative liée à une diminution du taux d'anticipation en condition Off $\mathrm{t}=2.39, \mathrm{p}=0.03$

Au total, les parkinsoniens avaient un taux d'anticipations et d'omissions plus élevé que les sujets témoins. De plus le taux d'anticipation chez les MP était plus élevé en condition On qu'en condition Off.

\section{Fréquence motrice}

La réciproque des fréquences motrices obtenues pour chaque groupe sont présentées dans le tableau $\mathbf{n}^{\circ} 7$.

\begin{tabular}{|l|l|l|}
\hline & MP & Témoins \\
\hline FMInv (ms) On & $242 \pm 77$ & $196 \pm 5,1$ \\
\hline FMInv (ms) Off & $272 \pm 95$ & $196 \pm 5,1$ \\
\hline
\end{tabular}

Tableau $n^{\circ} 7$ : Données de la réciproque des fréquences motrices (FMInv) exprimée en moyenne \pm écart-type. MP : maladie de Parkinson, ms : millisecondes.

La comparaison des groupes (MP et témoins) par un test $\mathrm{t}$ a montré une différence significative de la réciproque de la fréquence motrice des sujets parkinsoniens par rapport aux témoins liée à un ralentissement plus important chez les parkinsoniens $(\mathrm{t}=3,46 ; \mathrm{p}=0,0009)$. Puis un test t par série appariée a comparé les parkinsoniens en conditions $O n$ et $O f f$ et a mis en évidence un ralentissement significatif en condition $\operatorname{Off}(\mathrm{t}=-3.37 ; \mathrm{p}=0.002)$. 
Ces résultats montrent un ralentissement de la fréquence motrice chez les parkinsoniens comparativement aux témoins. Il existe également un ralentissement plus important de la fréquence motrice des parkinsoniens en condition Off par rapport à la condition On.

\section{Temps de réaction à choix}

Les résultats aux TRC moyens et le calcul de la latence décisionnelle sont présentés dans le tableau $\mathbf{n}^{\circ} 8$.

\begin{tabular}{|l|l|l|}
\hline & MP & Témoins \\
\hline TRC moyen (ms) On & $647 \pm 146$ & $595 \pm 105$ \\
\hline TRC moyen (ms) Off & $696 \pm 184$ & $595 \pm 105$ \\
\hline TRS moyen & 340 & 318 \\
\hline TRC moyen- TRS moyen (ms) & 307 & 278 \\
\hline
\end{tabular}

Tableau $\mathrm{n}^{\circ} 8$ : Performance des sujets aux temps de réaction à choix et calcul de la latence décisionnelle. Données exprimées en moyenne \pm écart-type. MP : maladie de Parkinson, ms : millisecondes.

L'analyse du TRC moyen a utilisé un test t avec le facteur intergroupe (MP et témoins), et intragroupe (condition $O n$ et $O f f$ ). La comparaison des groupes (MP et témoins) par un test t n'a pas montré de différence significative sur le TRC moyen $(\mathrm{t}=1,7 ; \mathrm{p}=0,09)$. Puis un test t par série appariée a comparé le TRC moyen des sujets parkinsoniens en condition $O n$ et $O f f$, et a montré une augmentation significative du TRC en condition Off par rapport à la condition $O n$ $(\mathrm{t}=-3,0 ; \mathrm{p}=0,004)$.

Un test t portant sur la latence décisionnelle (TRC moyen - TRS moyen) n'a pas montré pas de différence significative du temps décisionnel entre les patients et les témoins, $(\mathrm{t}=-1,0$; $\mathrm{p}=0,33)$. 
Au total, l'analyse des TRC n'a pas mis en évidence de différence entre les TRC moyens entre les deux groupes MP et témoins même s'il existe une tendance non significative à l'allongement chez les sujets parkinsoniens. De même que la latence décisionnelle n'est pas significativement augmentée chez les patients parkinsoniens par rapport aux témoins. Il existe un allongement significatif des TRC moyens des parkinsoniens en condition Off par rapport à la condition On.

Les principaux résultats obtenus peuvent être résumés de la façon suivante : on observe chez les sujets MP: (1) un ralentissement de la fréquence motrice en condition On majorée en condition Off ; (2) un ralentissement des temps d'inspection visuelle, sans effet On/Off ; (3) une préservation des TRS malgré un taux d'anticipation et d'omission supérieur ; (4) une préservation des TRC sans allongement de la latence décisionnelle. 


\section{DISCUSSION}

De même que Johnson [32], nous avons montré un ralentissement du TIV chez les sujets parkinsoniens suggérant un ralentissement du processus perceptif dans le ralentissement de l'action. Ballanger et al. [62] retrouvaient les mêmes conclusions au moyen d'un paradigme informatisé différent, ils utilisaient pour leur part, un stimulus cible dont la lisibilité était plus ou moins dégradée à chaque apparition, les résultats montraient une augmentation de la latence de la réponse motrice chez les sujets parkinsoniens par rapport à la population témoins. Ces résultats confirment les données de Shipley et al. [33] et de Sawomoto et al. [63] qui évoquaient un déficit significatif des procédés de perception dans la MP. Notre $2^{\text {ème }}$ analyse comparant les TIV des sujets parkinsoniens en condition $O n$ et $O f f$ ne montrait pas de différence significative, ainsi les procédés perceptifs déficitaires dans la MP utiliseraient, entre autres, des circuits non dopaminergique, confirmant la mise en jeu d'autres neuromédiateurs dans le ralentissement de l'action dans la MP.

Comme l'on pouvait s'y attendre la fréquence motrice, test permettant l'analyse du processus moteur dans le temps de réaction était augmenté dans la MP, évoquant un ralentissement du processus moteur, processus moteur évidemment dopa-sensible, comme le confirme les résultats comparant les parkinsoniens en condition $O n$ et $O f f$. Cependant de façon un peu surprenante les résultats des TRS (test également utilisé pour l'analyse des processus moteur), n'ont pas montré de ralentissement significatif des parkinsoniens par rapport aux témoins. Ce résultat peut être expliqué par le fait que le test mesurant les TRS, analyse à la fois le temps de la réponse motrice, et l'attention focalisée mis en jeu dans le temps de réaction, mais aussi, dans une moindre mesure, le temps du traitement perceptif (repérage du stimulus), et du traitement décisionnel (choix d'appuyer ou pas sur la cible), dans une forme assez élémentaire et basique pour ces deux derniers processus. Ainsi on peut se demander si le manque de spécificité de ce test, qui finalement est le reflet de l'ensemble des différents processus du temps de réaction à des degrés d'implication variable, peut expliquer l'absence de différence significative entre les parkinsoniens et les témoins. Et par ailleurs, comme vont le montrer les tests suivants, ces premiers résultats permettent déjà d'évoquer une relative préservation des capacités attentionnelles mises en jeu dans le TRS.

De nombreux travaux ont montré un ralentissement des TRS dans la MP, cependant la plupart des tests étaient passé dans les conditions habituels de traitements, dans notre étude l'analyse de comparaison entre sujets parkinsoniens et témoins s'est faite en condition On pour 
les parkinsoniens. Ainsi l'absence de ralentissement significatif retrouvé dans notre étude peut être liée à cette condition optimale dans laquelle se trouve le patient parkinsonien. A partir de ces résultats on peut penser que l'analyse des TRS en condition On pourrait être intéressante pour discriminer une MP des autres syndromes parkinsoniens plus, peu dopa-sensibles.

Par ailleurs, Stelmach et al [6] avait montré, que le ralentissement du TRS, entre parkinsoniens et témoins, était moins important lorsque le stimulus cible était précédé d'un signal avertisseur permettant aux sujets de se préparer à la réalisation du mouvement. Dans notre étude le signal avertisseur était séparé du stimulus cible à des intervalles variables allant de 350 à $600 \mathrm{~ms}$, et le mouvement réalisé était identique tout au long de la durée de l'épreuve, nos résultats peuvent ainsi être expliqué par cette capacité de pré-programmation qui ne semble pas être altérée dans la maladie de Parkinson. En effet Jahanshahi [10], avait confirmé que la capacité de pré-programmation était conservée dans la MP.

Par ailleurs on notera assez étonnamment que, le TRS au centile 5 était plus court chez les sujets parkinsoniens par rapport aux témoins, ce résultat peut être expliqué par le taux d'anticipation qui était significativement augmenté chez les parkinsoniens, ainsi les TRS les plus courts, sont probablement plus le fait de réponses anticipées que de réponses plus rapides des parkinsoniens.

L'analyse des TRC, qui permet de repérer un ralentissement des processus décisionnels, ne retrouve pas de ralentissement significatif chez les parkinsoniens, de même que la latence décisionnelle est comparable entre les deux groupes (MP et témoins). Des études antérieures retrouvaient des résultats similaires pour les TRC, alors même que, dans leurs analyses les TRS étaient ralentis chez les parkinsoniens. Dans notre étude les TRS et les TRC ne sont pas ralentis et le taux d'erreurs est similaire entre les deux groupes suggérant une préservation des processus décisionnels dans la MP. Nos résultats différent d'autres études notamment celle de Cooper [64], qui retrouvait chez 100 parkinsoniens, un allongement des TRC, cependant le paradigme employé faisait intervenir des processus perceptifs au $1^{\mathrm{er}}$ plan, en effet l'allongement du TRC était d'autant plus important que le stimulus cible se complexifiait, on peut ainsi se demander si le ralentissement retrouvé n'était pas lié plutôt à un déficit des processus perceptifs, comme nous l'avons démontré précédemment, plutôt qu'à un déficit des processus décisionnels.

Par ailleurs, nous n'avons pas mis en évidence de différence entre les témoins et les patients parkinsoniens au niveau de l'attention divisée, tant sur le nombre d'erreurs que sur le ralentissement. Ce résultat, ajoutait à l'analyse des TRS, qui étudie entre autre l'attention focalisée suggère une préservation des ressources attentionnelles. 
Ces résultats s'intègrent dans une littérature divergente quand à l'origine du ralentissement de l'action chez les parkinsoniens toutefois la population inclus dans cette étude correspond à celle décrite dans la littérature avec une prédominance des troubles dysexécutifs et un ralentissement objectivés par les échelles comportementales.

Ces divergences obtenues dans les différentes études peuvent s'expliquer par l'utilisation de paradigmes différents puisque jusqu'à présent aucun protocole standardisé n'existait. Mais également par l'inclusion de patients à des stades différents de la maladie, de durée d'évolution variable, de faible cohorte (longueur du temps de passation des protocoles), l'absence d'exclusion des sujets dépressifs alors que l'on sait que le syndrome dépressif allonge les temps de réaction. De ce fait, les comparaisons entre toutes ces études se doivent d'être analysées avec précaution.

Même si les données de la littérature sont contradictoires, notre étude s'appuie sur un paradigme de chronométrie standardisée et validée apportant une certaine solidité aux résultats présentés. Ainsi notre étude vient confirmer que le ralentissement de l'action concerne bien des processus spécifiques, à savoir les processus moteur et perceptif, et n'est pas lié à un ralentissement général. Aucune autre étude n'avait jusqu'à présent étudié dans un même temps les différents aspects du traitement de l'information: motrice, visuelle, attentionnelle et décisionnelle et cela au moyen d'un paradigme standardisé et validé. 


\section{CONCLUSION}

L'objectif de notre étude était d'analyser le ralentissement de l'action chez des patients parkinsoniens au moyen d'un paradigme de chronométrie mentale informatisé validé, et ainsi d'en préciser le mécanisme pour en dégager un profil. Notre travail a permis de mettre en évidence un profil discriminant du ralentissement de l'action montrant ainsi que les patients parkinsoniens ont une atteinte des processus moteurs et perceptifs.

Afin d'identifier des profils de ralentissement du temps de réaction spécifique à chaque syndrome parkinsonien, il pourrait être intéressant d'appliquer ce paradigme à d'autres pathologies, afin d'utiliser ces paramètres comme critère discriminatif entre les différents syndromes parkinsoniens. En effet, dans l'étude de Bailon [1], ce paradigme avait été employé dans la DCL et il apparaissait que les TRS et TRC étaient allongés de façon significative, alors que dans notre étude ces mêmes tests n'étaient pas allongés dans la MP.

L'utilisation de la chronométrie mentale pourrait donc devenir un outil supplémentaire au diagnostic différentiel entre les différents syndromes parkinsoniens où à des stades précoces les critères cliniques et paracliniques usuels peuvent échouer. 


\section{RÉFÉRENCES BIBLIOGRAPHIQUES}

1. Bailon O, Roussel M, Boucart M, Krystkowiak P, Godefroy O. Psychomotor slowing in mild cognitive impairment, Alzheimer's disease and lewy body dementia: mechanisms and diagnostic value. Dementia and geriatric cognitive disorders. 2010;29(5):388-96.

2. Godefroy O, Spagnolo S, Roussel M, Boucart M. Stroke and action slowing: mechanisms, determinants and prognosis value. Cerebrovascular diseases. 2010;29(5):508-14.

3. Stoquart-Elsankari S, Bottin C, Roussel-Pieronne M, Godefroy O. Motor and cognitive slowing in multiple sclerosis: an attentional deficit? Clinical neurology and neurosurgery. 2010 Apr;112(3):22632.

4. Godefroy O, Roussel M, Despretz P, Quaglino V, Boucart M. Age-related slowing: perceptuomotor, decision, or attention decline? Experimental aging research. 2010 Apr;36(2):169-89.

5. Bloxham CA, Mindel TA, Frith CD. Initiation and execution of predictable and unpredictable movements in Parkinson's disease. Brain : a journal of neurology. 1984 Jun;107 ( Pt 2):371-84.

6. Stelmach GE, Worringham CJ, Strand EA. Movement preparation in Parkinson's disease. The use of advance information. Brain : a journal of neurology. 1986 Dec;109 ( Pt 6):1179-94.

7. Benecke R, Rothwell JC, Dick JP, Day BL, Marsden CD. Disturbance of sequential movements in patients with Parkinson's disease. Brain : a journal of neurology. 1987 Apr;110 ( Pt 2):361-79.

8. Pullman SL, Watts RL, Juncos JL, Chase TN, Sanes JN. Dopaminergic effects on simple and choice reaction time performance in Parkinson's disease. Neurology. 1988 Feb;38(2):249-54.

9. Camicioli RM, Wieler M, de Frias CM, Martin WR. Early, untreated Parkinson's disease patients show reaction time variability. Neuroscience letters. 2008 Aug 15;441(1):77-80.

10. Jahanshahi M, Brown RG, Marsden CD. A comparative study of simple and choice reaction time in Parkinson's, Huntington's and cerebellar disease. Journal of neurology, neurosurgery, and psychiatry. 1993 Nov;56(11):1169-77.

11. Girotti F, Marano R, Soliveri P, Geminiani G, Scigliano G. Relationship between motor and cognitive disorders in Huntington's disease. Journal of neurology. 1988 Nov;235(8):454-7.

12. Posner MI, Petersen SE. The attention system of the human brain. Annual review of neuroscience. 1990;13:25-42.

13. Norman DA ST. Attention to action: willed and automatic control of behavior. Center for human information processing. Tecnical report. 1980;99.

14. Elbaz A, Bower JH, Maraganore DM, McDonnell SK, Peterson BJ, Ahlskog JE, et al. Risk tables for parkinsonism and Parkinson's disease. Journal of clinical epidemiology. 2002 Jan;55(1):25-31.

15. Pirozzolo FJ, Hansch EC, Mortimer JA, Webster DD, Kuskowski MA. Dementia in Parkinson disease: a neuropsychological analysis. Brain and cognition. 1982 Jan;1(1):71-83.

16. Muslimovic D, Post B, Speelman JD, Schmand B. Cognitive profile of patients with newly diagnosed Parkinson disease. Neurology. 2005 Oct 25;65(8):1239-45. 
17. Van Spaendonck KP, Berger HJ, Horstink MW, Buytenhuijs EL, Cools AR. Executive functions and disease characteristics in Parkinson's disease. Neuropsychologia. 1996 Jul;34(7):617-26.

18. Dujardin K, Tard C, Duhamel A, Delval A, Moreau C, Devos D, et al. The pattern of attentional deficits in Parkinson's disease. Parkinsonism \& related disorders. 2013 Mar;19(3):300-5.

19. Rodriguez-Ferreiro J, Cuetos F, Herrera E, Menendez M, Ribacoba R. Cognitive impairment in Parkinson's disease without dementia. Movement disorders : official journal of the Movement Disorder Society. 2010 Oct 15;25(13):2136-41.

20. Cools R. Dopaminergic modulation of cognitive function-implications for L-DOPA treatment in Parkinson's disease. Neuroscience and biobehavioral reviews. 2006;30(1):1-23.

21. Brown RG, Marsden CD. Internal versus external cues and the control of attention in Parkinson's disease. Brain : a journal of neurology. 1988 Apr;111 ( Pt 2):323-45.

22. Downes JJ, Roberts AC, Sahakian BJ, Evenden JL, Morris RG, Robbins TW. Impaired extradimensional shift performance in medicated and unmedicated Parkinson's disease: evidence for a specific attentional dysfunction. Neuropsychologia. 1989;27(11-12):1329-43.

23. Sharpe MH. Distractibility in early Parkinson's disease. Cortex; a journal devoted to the study of the nervous system and behavior. 1990 Jun;26(2):239-46.

24. Jordan N, Sagar HJ, Cooper JA. Cognitive components of reaction time in Parkinson's disease. Journal of neurology, neurosurgery, and psychiatry. 1992 Aug;55(8):658-64.

25. Evarts EV, Teravainen H, Calne DB. Reaction time in Parkinson's disease. Brain : a journal of neurology. 1981 Mar;104(Pt 1):167-86.

26. Harrison J, Henderson L, Kennard C. Abnormal refractoriness in patients with Parkinson's disease after brief withdrawal of levodopa treatment. Journal of neurology, neurosurgery, and psychiatry. 1995 Nov;59(5):499-506.

27. Rafal RD, Posner MI, Walker JA, Friedrich FJ. Cognition and the basal ganglia. Separating mental and motor components of performance in Parkinson's disease. Brain : a journal of neurology. 1984 Dec;107 ( Pt 4):1083-94.

28. Michely J, Barbe MT, Hoffstaedter F, Timmermann L, Eickhoff SB, Fink GR, et al. Differential effects of dopaminergic medication on basic motor performance and executive functions in Parkinson's disease. Neuropsychologia. 2012 Aug;50(10):2506-14.

29. Starkstein SE, Esteguy M, Berthier ML, Garcia H, Leiguarda R. Evoked potentials, reaction time and cognitive performance in on and off phases of Parkinson's disease. Journal of neurology, neurosurgery, and psychiatry. 1989 Mar;52(3):338-40.

30. Ogden J GJ, Corkin S. Deficits on visuospatial tests involving forward planning in highfunctioning Parkinsonians. Neuropsychiatry, Neuropsychology and Behavioral Neurology 1990;3:125-39.

31. McDowell SA, Harris J. Irrelevant peripheral visual stimuli impair manual reaction times in Parkinson's disease. Vision research. 1997 Dec;37(24):3549-58.

32. Johnson AM, Almeida QJ, Stough C, Thompson JC, Singarayer R, Jog MS. Visual inspection time in Parkinson's disease: deficits in early stages of cognitive processing. Neuropsychologia. 2004;42(5):577-83. 
33. Shipley BA, Deary IJ, Tan J, Christie G, Starr JM. Efficiency of temporal order discrimination as an indicator of bradyphrenia in Parkinson's disease: the inspection time loop task. Neuropsychologia. 2002;40(8):1488-93.

34. Giaschi D, Lang A, Regan D. Reversible dissociation of sensitivity to dynamic stimuli in Parkinson's disease: is magnocellular function essential to reading motion-defined letters? Vision research. 1997 Dec;37(24):3531-4.

35. Phillips JG, Schiffter T, Nicholls ME, Bradshaw JL, Iansek R, Saling LL. Does old age or Parkinson's disease cause bradyphrenia? The journals of gerontology Series A, Biological sciences and medical sciences. 1999 Aug;54(8):M404-9.

36. Malapani C, Pillon B, Dubois B, Agid Y. Impaired simultaneous cognitive task performance in Parkinson's disease: a dopamine-related dysfunction. Neurology. 1994 Feb;44(2):319-26.

37. Brown RG, Marsden CD. Dual task performance and processing resources in normal subjects and patients with Parkinson's disease. Brain : a journal of neurology. 1991 Feb;114 ( Pt 1A):215-31.

38. Dalrymple-Alford JC, Kalders AS, Jones RD, Watson RW. A central executive deficit in patients with Parkinson's disease. Journal of neurology, neurosurgery, and psychiatry. 1994 Mar;57(3):360-7.

39. O'Shea S, Morris ME, Iansek R. Dual task interference during gait in people with Parkinson disease: effects of motor versus cognitive secondary tasks. Physical therapy. 2002 Sep;82(9):888-97.

40. Hoehn MM, Yahr MD. Parkinsonism: onset, progression and mortality. Neurology. 1967 May;17(5):427-42.

41. Kalafat M H-DL, Poitrenaud J. Standardisation et étalonnage français du « Mini Mental State » (MMS) version GRECO. Neuropsychology review. 2003;13(2):209-36.

42. Fahn S, Elton RL and members of the UPDRS development committee. Unified Parkinson's Disease Rating Scale (UPDRS). In: Fahn S, Marsden CD, Calne DB, Goldstein M(Eds). Recent developments in Parkinson's disease. Florahm Park, NJ: Macmillan Health Care Information 1987; 3: 153-164.

43. Kehagia AA, Barker RA, Robbins TW. Neuropsychological and clinical heterogeneity of cognitive impairment and dementia in patients with Parkinson's disease. The Lancet Neurology. 2010 Dec;9(12):1200-13.

44. Cockrell JR FM. Mini-Mental State Examination (MMSE).Psychopharmacol Bull. 1988;24(4):689-92

45. Mattis S. Mental status examination for organic mental syndrome in the elderly patients. In Bellak L, Katasu TB (Eds.), Geriatric Psychiatry: A handbook for psychiatrists and primary care physicians 1976; 77-121. New York: Grune and Statton.

46. De Renzi E, Faglioni P. Normative data and screening power of a shortened version of the Token Test. Cortex; a journal devoted to the study of the nervous system and behavior. 1978 Mar;14(1):41-9.

47. Deloche G HD. Test de dénomination orale d'images. Les editions du centre de Psychologie Appliquée 1989.

48. Cardebat D, Doyon B, Puel M, Goulet P, Joanette Y. Formal and semantic lexical evocation in normal subjects. Performance and dynamics of production as a function of sex, age and educational level. Acta neurologica Belgica. 1990;90(4):207-17. 
49. Albert ML. A simple test of visual neglect. Neurology. 1973 Jun;23(6):658-64.

50. Agniel A, Joanette, T., Doyon, B., \& Duchein, C. Protocole Montréal Toulouse d'évaluation des gnosies visuelles. Isbergues, France: L'ortho-Édition. 1992.

51. Rey A. Test de copie d'une figure complexe. Les éditions du Centre de Psychologie Appliquée 1959.

52. Grober E, Buschke H. Genuine memory deficits in dementia. Developmental Neuropsychology 1987; 3: 13-36.

53. Van Der Linden M, Coyette F, Poitrenaud J, Kalafat M, Calacis F, Wyns C, Adam S: L'épreuve de rappel libre/rappel indicé à 16 items; in Van Der Linden M, Adam S, Agniel A, Baisset Mouly C (eds): L'évaluation des troubles de la mémoire: présentation de quatre tests de mémoire épisodique (avec leur étalonnage). Marseille, Solal, 2004, pp 25-48.

54. Reitan RM. Validity of trail making tests as an indicator of organic brain injury. Baltimore; Johns Hopkins University Press 1986.

55. Nelson HE. A modified card sorting test sensitive to frontal lobe defects. Cortex; a journal devoted to the study of the nervous system and behavior. 1976 Dec;12(4):313-24.

56. P.W. Burgess TS. The Hayling and Brixton Tests. Thames Valley Test Company, Thurston, Suffolk. 1997.

57. J Stroop JR. Studies of interference in serial verbal reactions. J Exp Psychol 1935; 18: 643-662.

58. Montgomery SA, Asberg M. A new depression rating scale designed to be sensitive to change. $\mathrm{Br}$ J Psychiatry 1979; 134: 382-389.

59. Godefroy O AP, Robert P, Roussel M,. Dysexecutive syndrome: diagnostic criteria and validation study. 2009.

60. Godefroy O, Lhullier-Lamy C, Rousseaux M. SRT lengthening: role of an alertness deficit in frontal damaged patients. Neuropsychologia 2002; 40: 2234-2241.

61. Godefroy O, Rousseaux M. Binary choice in patients with prefrontal or posterior brain damage. A relative judgement theory analysis. Neuropsychologia 1996; 34: 1029-1038.

62. Ballanger B, Gil R, Audiffren M, Desmurget M. Perceptual factors contribute to akinesia in Parkinson's disease. Experimental brain research. 2007 May;179(2):245-53.

63. Sawamoto N, Honda M, Hanakawa T, Fukuyama H, Shibasaki H. Cognitive slowing in Parkinson's disease: a behavioral evaluation independent of motor slowing. The Journal of neuroscience : the official journal of the Society for Neuroscience. 2002 Jun 15;22(12):5198-203.

64. Cooper JA, Sagar HJ, Tidswell P, Jordan N. Slowed central processing in simple and go/no-go reaction time tasks in Parkinson's disease. Brain : a journal of neurology. 1994 Jun;117 ( Pt 3):517-29. 
ANNEXE $\mathbf{N}^{\circ} 1$

\begin{tabular}{|c|c|}
\hline Drug & Conversion factor \\
\hline Immediate release L-dopa & $\mathbf{x} 1$ \\
\hline Controlled release L-dopa & $\times 0.75$ \\
\hline Entacapone (or Stalevo®) & LD $\times 0.33$ \\
\hline Tolcapone & LD $\times 0.5$ \\
\hline Duodopa ${ }^{\circledR}$ & X1.11 \\
\hline Pramipexole (as salt) & $\times 100$ \\
\hline Ropinirole & X 20 \\
\hline Rotigotine & X 30 \\
\hline Selegiline-Oral & X 10 \\
\hline Selegiline - sublingual & $\times 80$ \\
\hline Rasagiline & $\times 100$ \\
\hline Amantadine & $\mathbf{x} 1$ \\
\hline Apomorphine & $\times 10$ \\
\hline
\end{tabular}

Tableau de conversion des traitements dopaminergiques en dose équivalent L-Dopa selon Tomlinson CL, Stowe R, Patel S, Rick C, Gray R, Clarke CE. Systematic review of levodopa dose equivalency reporting in Parkinson's disease. Movement disorders : official journal of the Movement Disorder Society. 2010 Nov 15;25(15):2649-53. 


\begin{abstract}
BACKGROUND : The action slowing in Parkinson's disease (PD) has been investigated in several studies, nevertheless with contradictory results. The using of different methods can explain these divergences. Therefore it is difficult to compare them. It was related that action slowing in PD is not only because of motor deficit but both combining motor and cognition slowing. Two methods enable to explore the action slowing and attention deficit: neuropsychological tests and chronometry mental tests. Reaction time tests enable more specific and quantitative description of action slowing and attentional deficit.
\end{abstract}

OBJECTIVES : The aim of our study was first to specify action slowing in PD and secondly to identify the mecanisms by means of computerized and validated chronometry's paradigm.

METHODS : Fourty patients with PD from stage 1 to 3 of Hoen and Yahr and fourty controls matched on age and level of education were included. Each individual carried out a whole battery of tests containing finger tapping, simple reaction time in simple and double condition, choice reaction time and visual inspection time tasks. These tasks were first conducted on PD group in condition Off which means before their dopaminergic treatment and secondly in condition $O n$ : 1H30 after a supraliminal dose of L-Dopa.

RESULTS : We noticed the PD's group had (1) lower motor tapping frequency in condition $O n$ ( $\mathrm{p}=0.0009)$, which raised in condition Off ; (2) the simple reaction time was maintained $(\mathrm{p}=0.8)$, although (3) the superior rate of anticipation in PD's group ( $\mathrm{p}=0.04)$, (4) a slowdown in double condition which not really differed from the control, (5) the choice reaction time and decision latency were also maintained $(\mathrm{p}=0.09),(6)$ and finally an increase of visual inspection time $(\mathrm{p}=0.02)$, not dopa-responsive.

CONCLUSIONS : Our study showed that action slowing in PD is mainly related to slower perceptuomotor processes, whereas decisional and attentional processes are preserved.

Keywords : Parkinson's disease, Reaction time, Perception, Attention, Action slowing 


\section{RÉSUMÉ}

INTRODUCTION : Le ralentissement de l'action dans la maladie de Parkinson (MP) a été étudié dans de nombreuses études mais avec des résultats parfois contradictoires. Deux méthodes permettent d'explorer le ralentissement de l'action et le déficit attentionnel: les tests neuropsychologiques et les tests de chronométrie mentale. Les tests de temps de réaction permettent une description quantitative et plus spécifique du ralentissement de l'action et du déficit attentionnel.

OBJECTIF : L'objectif de notre étude était de confirmer le ralentissement de l'action dans la MP, au moyen d'une batterie de tests de chronométrie informatisée, validée et standardisée, et d'identifier un profil discriminant du ralentissement de l'action.

MÉTHODE : Quarante patients MP et quarante témoins appariés en âge et au niveau d'éducation ont été inclus. Chacun des sujets a effectué une batterie de tests comprenant une tâche de fréquence motrice, de temps de réaction simple en condition simple et double, de temps de réaction à choix et de temps d'inspection visuel. Pour les sujets MP, les tests étaient réalisés d'abord en condition $O f f$ soit à jeun de leur traitement dopaminergique puis en condition $O n$ soit 1 h30 après la prise d'une dose supraliminaire de L-Dopa. Des analyses de comparaison de groupe (test $t$ de Student, MANOVA) ont été réalisées.

RÉSULTATS : Les résultats des tests chronométriques montrent chez les sujets MP (1) un ralentissement de la FM en condition On ( $\mathrm{p}=0.0009)$, majorée en condition Off; (2) une préservation des TRS ( $\mathrm{p}=0.8$ ), malgré un taux d'anticipation supérieur chez les MP par rapport aux témoins ( $\mathrm{p}=0.04)$; (3) un ralentissement en condition duelle qui ne différait toutefois pas des témoins ; (4) une préservation des TRC ( $\mathrm{p}=0.09)$; (5) et enfin un allongement des TIV ( $\mathrm{p}=0.02)$, non dopa-sensible $(\mathrm{p}=0.4)$.

CONCLUSION : Notre travail a donc objectivé un ralentissement de l'action chez les parkinsoniens caractérisé essentiellement par une atteinte des processus perceptivo-moteurs, et une préservation des processus décisionnels et attentionnels.

Mots clés : Maladie de Parkinson, Ralentissement de l'action, Perception, Attention 


\section{ÉTUDE DU RALENTISSEMENT DE L'ACTION DANS LA MALADIE DE PARKINSON AU MOYEN D'UN PARADIGME DE CHRONOMÉTRIE INFORMATISÉE}

\section{RÉSUMÉ}

INTRODUCTION : Le ralentissement de l'action dans la maladie de Parkinson (MP) a été étudié dans de nombreuses études mais avec des résultats parfois contradictoires. Deux méthodes permettent d'explorer le ralentissement de l'action et le déficit attentionnel : les tests neuropsychologiques et les tests de chronométrie mentale. Les tests de temps de réaction permettent une description quantitative et plus spécifique du ralentissement de l'action et du déficit attentionnel.

OBJECTIF : L'objectif de notre étude était de confirmer le ralentissement de l'action dans la MP, au moyen d'une batterie de tests de chronométrie informatisée, validée et standardisée, et d'identifier un profil discriminant du ralentissement de l'action.

MÉTHODE : Quarante patients MP et quarante témoins appariés en âge et au niveau d'éducation ont été inclus. Chacun des sujets a effectué une batterie de tests comprenant une tâche de fréquence motrice, de temps de réaction simple en condition simple et double, de temps de réaction à choix et de temps d'inspection visuel. Pour les sujets MP, les tests étaient réalisés d'abord en condition Off soit à jeun de leur traitement dopaminergique puis en condition $O n$ soit $1 \mathrm{~h} 30$ après la prise d'une dose supraliminaire de L-Dopa. Des analyses de comparaison de groupe (test $t$ de Student, MANOVA) ont été réalisées.

RÉSULTATS : Les résultats des tests chronométriques montrent chez les sujets MP (1) un ralentissement de la FM en condition On ( $\mathrm{p}=0.0009)$, majorée en condition Off ; (2) une préservation des TRS $(\mathrm{p}=0.8)$, malgré un taux d'anticipation supérieur chez les MP par rapport aux témoins $(\mathrm{p}=0.04)$; (3) un ralentissement en condition duelle qui ne différait toutefois pas des témoins; (4) une préservation des TRC $(\mathrm{p}=0.09)$; (5) et enfin un allongement des TIV ( $\mathrm{p}=0.02$ ), non dopa-sensible $(\mathrm{p}=0.4)$.

CONCLUSION : Notre travail a donc objectivé un ralentissement de l'action chez les parkinsoniens caractérisé essentiellement par une atteinte des processus perceptivomoteurs, et une préservation des processus décisionnels et attentionnels.

Mots clés : Maladie de Parkinson, Ralentissement de l'action, Perception, Attention. 